\title{
Effect of Change in Systolic Blood Pressure Between Clinic Visits on Estimated 10-Year Cardiovascular Disease Risk
}

\author{
Siqin Ye, MD, MS ${ }^{1}$, Y. Claire Wang, MD, ScD², Daichi Shimbo, MD¹, Jonathan D. Newman, \\ MD, MPH ${ }^{1}$, Emily B. Levitan, $\mathbf{S c D}^{3}$, and Paul Muntner, $\mathbf{P h D}^{3}$ \\ ${ }^{1}$ Center for Behavioral Cardiovascular Health, Department of Medicine, Columbia University, New \\ York, New York \\ ${ }^{2}$ Department of Health Policy \& Management, Mailman School of Public Health, Columbia \\ University, New York, New York \\ ${ }^{3}$ Department of Epidemiology, University of Alabama at Birmingham, Birmingham, Alabama
}

\begin{abstract}
Background-Systolic blood pressure (SBP) often varies between clinic visits within individuals, which can affect estimation of cardiovascular disease (CVD) risk.

Methods and Results-We analyzed data from participants with two clinic visits separated by a median of 17 days in the Third National Health and Nutrition Examination Survey $(\mathrm{n}=808)$. Tenyear CVD risk was calculated with SBP obtained at each visit using the Pooled Cohort Equations. The mean age of participants was 46.1 years and $47.3 \%$ were male. The median SBP difference between the two visits was $-1 \mathrm{mmHg}\left(1^{\text {st }}-99^{\text {th }}\right.$ percentiles: -23 to $\left.32 \mathrm{mmHg}\right)$. The median estimated 10 -year CVD risk was $2.5 \%$ and $2.4 \%$ at the first and second visit, respectively $\left(1^{\text {st }}-\right.$ $99^{\text {th }}$ percentiles $-5.2 \%$ to $+7.1 \%$ ). Meaningful risk reclassification (i.e., across the guideline recommended $7.5 \%$ threshold for statin initiation) occurred in $12(11.3 \%)$ of 106 participants whose estimated CVD risk was between $5 \%$ to $10 \%$, but only in $2(0.3 \%)$ of 702 participants who had a 10-year estimated CVD risk of $<5 \%$ or $>10 \%$.
\end{abstract}

Conclusions-SBP variability can affect CVD risk estimation, and can influence statin eligibility for individuals with an estimated 10-year CVD risk between 5\% and 10\%.

\section{Keywords}

systolic blood pressure; risk assessment; statins; adults

\section{Introduction}

The newly published American College of Cardiology (ACC)/ American Heart Association (AHA) Guideline on the Treatment of Blood Cholesterol to Reduce Atherosclerotic Cardiovascular Risk in Adults recommend estimating 10-year cardiovascular disease (CVD)

(C) 2013 American Society of Hypertension. Published by Elsevier Inc. All rights reserved Correspondence: Siqin Ye, MD Columbia University Medical Center 622 West $168^{\text {th }}$ Street, PH 9-320 New York, NY 10032, USA Phone: (212)-342-5633 Fax: (212)-342-3431 sy2357@ columbia.edu.

Publisher's Disclaimer: This is a PDF file of an unedited manuscript that has been accepted for publication. As a service to our customers we are providing this early version of the manuscript. The manuscript will undergo copyediting, typesetting, and review of the resulting proof before it is published in its final citable form. Please note that during the production process errors may be discovered which could affect the content, and all legal disclaimers that apply to the journal pertain.

Conflicts of interest: Other authors report no additional disclosures or conflicts of interest. 
risk using the Pooled Cohort Equations to guide initiation of statin therapy for primary prevention. $(1,2)$ Systolic blood pressure is one of the measures used in the calculation of estimated 10-year CVD risk.(1) Substantial change in systolic blood pressure between sequential clinic visits has been noted in research studies and in clinical practice.(3-6) For example, Rothwell et al. demonstrated that $15-35 \%$ of participants treated for hypertension or who had a prior transient ischemic attack had changes in systolic blood pressure greater than $50 \mathrm{~mm} \mathrm{Hg}$ across multiple clinic visits.(3) Therefore, systolic blood pressure variability has the potential to affect estimation of an individual's 10-year CVD risk, potentially leading to uncertainty for the classification of patients into risk categories intended to guide prevention efforts. However, scarce data are available on how likely this reclassification is to occur.

With the release of the 2013 ACC / AHA guidelines that emphasize absolute CVD risk for the initiation of statin therapy,(2) there is a need to characterize the impact of changes in systolic blood pressure across clinic visits on the stability of CVD risk estimation. To address this, we used data from the subset of participants in the Third National Health and Nutrition Examination Survey (NHANES III) who completed two clinic visits over a period of several weeks, to determine the effect of changes in systolic blood pressure across visits on changes in estimated 10-year CVD risk.

\section{Methods}

NHANES III was a cross-sectional survey designed to select a representative sample of the civilian non-institutionalized US population.(7) The protocol for NHANES III was approved by the National Center for Health Statistics of the Centers for Disease Control and Prevention Institutional Review Board, and all participants provided informed consent. Over the survey period of 1988-1994, 18,825 adults $\geq 20$ years of age completed an in-home interview and a visit to a mobile clinic (Visit 1) for medical evaluation including three blood pressure measurements. A subsample $(\mathrm{n}=2,174)$ attended a second visit at the mobile clinic (Visit 2), at which time the medical evaluation, including three blood pressure measurements, was repeated. The median duration between the two clinic visits was 17 days (range, 1 to 48 days). Although more recent NHANES are available, we chose to analyze NHANES III as it has data from a second study visit allowing for the calculation of variability of blood pressure across two study visits. For the current analyses, we included NHANES III participants who had two clinic visits with three blood pressure measurements performed in the same arm at each visit $(n=956)$. We excluded 32 participants missing data on measures (e.g., total cholesterol) needed to calculate estimated 10-year CVD risk, and 116 participants with a history of coronary heart disease (CHD), diabetes, or stroke, as they would be classified as having high CVD risk based on medical history alone.(2) After these exclusions, 808 participants were included in this analysis.

\section{Data Collection and Blood Pressure Measurement}

Demographic and health-related information including age, sex, race/ethnicity, and smoking status was collected using a standardized questionnaire during the in-home interview. The use of anti-hypertensive medications was ascertained via self-report. Blood specimens were collected during the medical evaluation to determine total cholesterol and high-density lipoprotein (HDL) cholesterol levels. Blood pressure was measured three times during each of the two clinic visits, with the average of the second and third measurements recorded as the blood pressure for that visit. Blood pressure measurements were performed by a trained clinician using the standard protocol of the American Heart Association at both clinic visits. Each participant's arm was measured to determine the appropriate sized cuff. Participants were asked to remain in a seated position for five minutes of quiet rest prior to their first blood pressure measurement being taken. Quality control for the blood pressure 
measurements included quarterly recertification with retraining if necessary, annual retraining of all physicians, and monitoring of equipment and equipment repair. Additional details regarding blood pressure measurement and quality control procedures are provided in the NHANES III manual of operations.(7)

\section{Statistical Analysis}

At each clinic visit, we calculated the participant's estimated 10 year CVD risk using the Pooled Cohort Equations based on age, sex, race, systolic blood pressure, total cholesterol, HDL cholesterol, smoking status, and use of antihypertensive medication.(1) In order to isolate the effect of systolic blood pressure variability on estimated 10-year CVD risk, we used the systolic blood pressure measured at each visit, while assuming other parameters (e.g., cholesterol levels, smoking status) remain unchanged from the first visit. We then computed the difference in 10-year CVD risk estimates calculated at the two visits, defined as the risk estimate from Visit 2 minus the risk estimate from Visit 1 . The summary measures for number of days between clinic visits, systolic blood pressure, diastolic blood pressure, and the estimated 10-year CVD risk at each visit were expressed as medians and $25^{\text {th }}$ to $75^{\text {th }}$ percentiles due to non-normal distributions of these variables. Agreement between the two 10-year CVD risk estimates was assessed using a Bland-Altman plot,(8) and the proportion of individuals with $\geq 1 \%, \geq 2 \%, \geq 3 \%, \geq 4 \%$, and $\geq 5 \%$ absolute change in estimated 10-year CVD risk across two clinic visits was calculated. Next, we examined the effect of change in blood pressure on change in estimated 10-year CVD risk across two visits. First, we calculated the percentage of participants with systolic blood pressure $<130$, 130 to $<150$, and $\geq 150 \mathrm{~mm} \mathrm{Hg}$ and diastolic blood pressure $<75,75$ to 84 and $\geq 85 \mathrm{~mm} \mathrm{Hg}$ at the first visit who experienced $23 \%$ absolute change in estimated 10-year CVD risk between Visit 1 and Visit 2; $23 \%$ was chosen to represent a change large enough to have potential clinical relevance. Using logistic regression models with systolic blood pressure < $130 \mathrm{~mm} \mathrm{Hg}$ as the reference group, we analyzed whether systolic blood pressure between 130 to $<150 \mathrm{~mm} \mathrm{Hg}$ and $\geq 150 \mathrm{~mm} \mathrm{Hg}$ at time of Visit 1 were associated with a $\geq 3 \%$ absolute change in estimated 10-year CVD risk at Visit 2 after multivariable adjustment. An initial model adjusted for age, sex, race-ethnicity, and a second model further included additional adjustment for diastolic blood pressure. Logistic regression models were also constructed for diastolic blood pressure ( $<75$ [reference], 75 to 84 and $\geq 85 \mathrm{~mm} \mathrm{Hg}$ ) with two levels of adjustment for (Model 1) age, sex, race-ethnicity and (Model 2) age, sex, raceethnicity and systolic blood pressure.

Using 10-year CVD risk categories of $<5 \%, 5 \%$ to $<7.5 \%, 7.5 \%$ to $<10 \%$, and $\geq 10 \%$, (2) we calculated the proportion of participants who were reclassified from Visit 1 to Visit 2. Specifically, we calculated the percentage of participants who stayed in the same riskcategory between Visits 1 and 2, were re-classified downwards (e.g., from $\geq 10 \%$ at Visit 1 to $7.5 \%$ to $<10 \%$ at Visit 2), or were re-classified upwards (e.g., from $7.5 \%$ to $<10 \%$ at Visit 1 to $\geq 10 \%$ at Visit 2). Examples of NHANES III participants whose risk categories changed due to change in systolic blood pressure across the two clinic visits are provided to illustrate the clinical implications of our findings. In a secondary analysis, we repeated the above analysis using the Framingham risk equation for estimated 10-year CHD risk from the Adult Treatment Panel III guidelines and the recommended risk categories of $<10 \%, 10 \%$ to $<20 \%$, and $220 \%$. $(9,10)$

All analyses were conducted without sampling weights as recommended for NHANES III second examination data, and were performed using the statistical package SAS version 9.2 (SAS Institute, Cary, NC). 


\section{Results}

Of the 808 participants included in the analyses, the mean (SD) age was 46.1 (17.2) years; $47.3 \%$ were men; $42.2 \%$ were non-Hispanic white; and $29.2 \%$ were current smokers (Table 1). The mean (SD) level for total cholesterol was $5.31(1.08) \mathrm{mmol} / \mathrm{L}$ and for HDL cholesterol was $1.31(0.39) \mathrm{mmol} / \mathrm{L}$. Overall, $14.5 \%$ of participants were taking antihypertensive medications.

\section{Blood Pressure and Estimated CVD Risk at Two Clinic Visits}

At Visit 1 , the median ( $25^{\text {th }}$ to $75^{\text {th }}$ percentiles) systolic blood pressure was 118 (108 to 131) $\mathrm{mm} \mathrm{Hg}$ (Table 2$)$, and the median $\left(25^{\text {th }}\right.$ to $75^{\text {th }}$ percentiles) estimated 10 -year CVD risk was $2.5 \%(0.5 \%$ to $10.0 \%)$. At Visit 2 , the median $\left(25^{\text {th }}\right.$ to $75^{\text {th }}$ percentiles $)$ systolic blood pressure was 117 (107 to 129) $\mathrm{mm} \mathrm{Hg}$, and the median $\left(25^{\text {th }}\right.$ to $75^{\text {th }}$ percentiles) estimated 10 -year CVD risk was $2.4 \%$ ( $0.5 \%$ to $10.0 \%)$. The median within-person differences in systolic blood pressure between Visit 1 and Visit 2 was $-1 \mathrm{~mm} \mathrm{Hg}\left(1^{\text {st }}\right.$ to $99^{\text {th }}$ percentiles, -23 to $32 \mathrm{mmHg}$ ). For individual participants, changes in systolic blood pressure from Visit 1 to Visit 2 resulted in a wide distribution of changes in estimated 10-year CVD risk (median $=0.0 \% ; 1^{\text {st }}$ to $99^{\text {th }}$ percentiles $-5.2 \%$ to $+7.1 \%$ ). Overall, $23 \%$ of participants had an absolute difference in estimated 10 -year CVD risk that was $\geq 1 \%$ across the 2 visits; $8 \%$ of participants had an absolute difference that was $23 \%$; and $4 \%$ of participants had an absolute difference that was $25 \%$ (Figure 1). On average, the difference in estimated 10-year CVD risk between the two visits was larger for individuals with higher estimated risk (Figure 2).

Overall, 2.9\% of participants with systolic blood pressure $<130 \mathrm{~mm} \mathrm{Hg}$ at Visit 1 had a $33 \%$ absolute change in estimated 10-year CVD risk between Visits 1 and 2, compared to $13.6 \%$ and $36.0 \%$ of participants with systolic blood pressure $130-149$ and $\geq 150 \mathrm{~mm} \mathrm{Hg}$, respectively, at Visit 1 (Table 3, top panel). After adjustment for age, gender, race-ethnicity, and diastolic blood pressure and compared to participants with systolic blood pressure $<130$ $\mathrm{mm} \mathrm{Hg}$ at Visit 1, the odds ratio for having a $23 \%$ absolute change in estimated 10-year CVD risk between Visits 1 and 2 associated with systolic blood pressure of 130-149 $\mathrm{mm} \mathrm{Hg}$ and $\geq 150 \mathrm{~mm} \mathrm{Hg}$ were 1.86 (95\% CI 0.84-4.12) and 5.60 (95\% CI 2.23-14.1), respectively. In contrast, no association was present between diastolic blood pressure categories and having a $\geq 3 \%$ between-visit absolute change in estimated 10-year CVD risk (Table 3, bottom panel).

\section{Reclassification Due to Changes in Blood Pressure}

For participants with an estimated 10-year CVD risk of $<5 \%$ at Visit $1,1.2 \%$ were reclassified upwards at Visit 2, including 1 participant who was reclassified into the $7.5 \%$ to $<10 \%$ category (Table 4 ). In contrast, $12.1 \%$ of participants who had an estimated 10-year CVD risk in the $5 \%$ to $<7.5 \%$ category at Visit 1 were reclassified downwards to the $<5 \%$ category, while $10.3 \%$ were re-classified upwards to the $7.5 \%$ to $<10 \%$ category. Similarly, $12.5 \%$ of participants with an estimated 10 -year CVD risk in the $7.5 \%$ to $<10 \%$ category at Visit 1 were reclassified downwards to the $5 \%$ to $<7.5 \%$ category and $10(20.8 \%)$ were reclassified upwards into the $\geq 10 \%$ category at Visit 2 . Finally, $4.5 \%$ of participants with estimated 10-year CVD risk of $\geq 10 \%$ at Visit 1 were reclassified downwards at Visit 2, including 1 participant who was reclassified into the $5 \%$ to $<7.5 \%$ category. Using the Framingham risk equation recommended in the ATP III guidelines and categories of $<10 \%$, $10 \%$ to $20 \%$, and $>20 \%$ of estimated 10-year CHD risk, reclassification from Visit 1 to Visit 2 due to change in systolic blood pressure primarily occurred in those in the highest estimated risk category (Table 5). 
We provide examples of two NHANES participants who were reclassified into different 10year CVD risk categories due to change in systolic blood pressure across two clinic visits in Table 6.

\section{Discussion}

In the current study, we demonstrated that change in systolic blood pressure between clinic visits could affect estimation of 10-year CVD risk using the recently published Pooled Cohort Equations. This effect was especially prominent in individuals with an estimated 10year CVD risk between 5\% and 10\%. However, reclassification in estimated 10-year CVD risk categories occurred in a very small percentage of individuals at low (i.e., $<5 \%$ ) or high (i.e., $\geq 10 \%$ ) estimated risk. The reclassification of risk associated with changes in systolic blood pressure between clinic visits is not unique to the Pooled Cohort Equations, as similar reclassification also occurred using the Framingham 10-year CHD risk equation.

Substantial blood pressure variability has been reported in previous studies. We have described a mean standard deviation of $7.7 \mathrm{~mm} \mathrm{Hg}$ for systolic blood pressure across three visits (two clinic visits and one home visit) in a subsample of NHANES III participants, as well as a mean standard deviation of $10.9 \mathrm{~mm} \mathrm{Hg}$ for systolic blood pressure measured across eleven visits in the Women's Health Initiative. $(4,11)$ Systolic blood pressure variability of a similar magnitude or greater has also been described in patients treated for hypertension or prior transient ischemic attack,(3) and in patients with hypertension followed in primary care clinics.(5) Our current analysis suggests that these levels of blood pressure variability $(3-5,11)$ can affect estimation of cardiovascular risk using the Pooled Cohort Equations recommended by current guidelines.(1) Especially for individuals whose estimated 10-year CVD risk is near the 7.5\% cut-point recommended for treatment decisions in the newly published ACC/AHA Guideline on the Treatment of Blood Cholesterol,(2) systolic blood pressure variability may result in uncertainty for clinical decisions such as statin initiation.

Although the current analysis focused on 10-year CVD risk estimated using the Pooled Cohort Equation, the issue of blood pressure variability is likely relevant to other methods of CVD and CHD risk prediction. As we have demonstrated, change in systolic blood pressure across clinic visits also affected the Framingham equation estimated 10-year CHD risk. Systolic blood pressure is also part of other commonly used risk prediction tools such as the Reynolds, the QRISK, and the SCORE risk scores,(12-14) and the stability of risk classification with these other methods may be affected by blood pressure variability. The increased emphasis on absolute risk in current national and international cardiovascular disease prevention guidelines $(2,15)$ suggest that that the stability of risk estimation is an issue that warrants further investigation.(16) Given the known challenges of applying risk prediction methods derived from populations to individual clinical encounters,(17) more study is also needed to determine whether and how to discuss the stability of risk classification during shared decision making processes.(18)

Our results also provide guidance for the appropriate use of Pooled Cohort Equations to estimate 10-year CVD risk in real-world settings. It is reassuring that we found change in systolic blood pressure across clinic visits meaningfully impacted estimation of 10-year CVD risk in only a small percentage of participants with 10-year CVD risk estimated to be $<5 \%$ or $\geq 10 \%$. These results suggest that systolic blood pressure measured at a single visit is sufficient when the estimated CVD risk is in these ranges. However, for individuals whose initial estimated risk is between 5\% to $10 \%$, we found that reclassification may occur for a substantial percentage of individuals due to change in systolic blood pressure. For these individuals, there is potential for over- or under-treatment with statin therapy if the clinical 
decision-making is guided solely by risk estimation at the initial visit. Further research is needed to clarify the extent to which repeat blood pressure measurements are needed to reliably estimate cardiovascular risk and to avoid under or over-treatment with statins.

Our study has several strengths. First, the NHANES data we used includes a broad crosssection of the US population, making our results highly generalizable. Second, blood pressure measurements in NHANES were performed by trained physicians following a standardized protocol, suggesting that the reclassification observed was due to within-person variability in blood pressure rather than measurement error. In addition, the two blood pressure measurements were taken only several weeks apart, making it less likely that our results are due to natural progression of hypertension or to changes in medication regimens. Taken together, our findings add to the emerging literature on the inherent challenges for applying population-derived risk prediction tools such as the Pooled Cohort Equations to individual clinical encounters. $(17,19)$

There are also several limitations to our study. Our sample size was limited by the number of NHANES III participants with two clinic visits, which represents only a subset of NHANES III cohort. However, the distribution of estimated cardiovascular risk in our sample is similar to what has been previously reported for the entire NHANES III cohort, (20) suggesting that our findings are not due to sampling or selection bias. As we calculated systolic blood pressure using the average of the second and third measurements at each visit, caution is also needed to generalize our results to clinical settings where blood pressure reading is often obtained through a single measurement without necessarily following a standardized protocol. However, systolic blood pressure measured in such settings will likely have higher variability across visits.(21) Finally, our analysis focused on risk estimation rather than hard clinical endpoints. However, our previous analysis of this study population also demonstrated that visit-to-visit variability of systolic blood pressure was associated with all-cause mortality, and was correlated with factors such as higher mean systolic blood pressure and the use of angiotensin converting enzyme-inhibitors.(4) Our results will need to be validated in future studies, and the clinical impact of uncertainty in risk estimation caused by systolic blood pressure variability will require further clarification.

In conclusion, we found that change in systolic blood pressure across clinic visits has a clinically meaningful effect on estimation of 10-year CVD risk using the Pooled Cohort Equations, especially for individuals whose estimated risk is near the $7.5 \%$ cut-point recommended for consideration of statin therapy. These results may be useful to guide interpretation of estimated 10-year CVD risk during individual clinical encounters where statin initiation is considered, and suggest that there is a need to better understand the effect of blood pressure variability on the stability of risk classification for risk prediction methods.

\section{Acknowledgments}

Funding and Support: Dr. Ye is supported by National Institutes of Health Grant T32 HL007854-16, and by National Center for Advancing Translational Sciences, National Institutes of Health, through Grant Number UL1 TR000040, formerly the National Center for Research Resources, Grant Number UL1 RR024156. Drs. Wang and Shimbo are supported by National Institute of Health Grant P01 HL47540. The content of this publication is solely the responsibility of the authors and does not necessarily represent the official views of the NIH.

Dr. Muntner has received grant funding and served as a consultant to Amgen Corporation. Dr. Levitan has received research support from Amgen Corporation. 


\section{References}

1. Goff DC, Lloyd-Jones DM, Bennett G, Coady S, D'Agostino RB, Gibbons R, et al. 2013 ACC/AHA Guideline on the Assessment of Cardiovascular Risk: A Report of the American College of Cardiology/American Heart Association Task Force on Practice Guidelines. Circulation. Nov 12.20132013.

2. Stone NJ, Robinson J, Lichtenstein AH, Merz CNB, Blum CB, Eckel RH, et al. 2013 ACC/AHA Guideline on the Treatment of Blood Cholesterol to Reduce Atherosclerotic Cardiovascular Risk in Adults: A Report of the American College of Cardiology/American Heart Association Task Force on Practice Guidelines. Circulation. Nov 12.2013 2013.

3. Rothwell PM, Howard SC, Dolan E, O'Brien E, Dobson JE, Dahlof B, et al. Prognostic significance of visit-to-visit variability, maximum systolic blood pressure, and episodic hypertension. Lancet. Mar 13; 2010 375(9718):895-905. PubMed PMID: 20226988. Epub 2010/03/17. eng. [PubMed: 20226988]

4. Muntner P, Shimbo D, Tonelli M, Reynolds K, Arnett DK, Oparil S. The relationship between visitto-visit variability in systolic blood pressure and all-cause mortality in the general population: findings from NHANES III, 1988 to 1994. Hypertension. Feb; 2011 57(2):160-6. PubMed PMID: 21200000. Epub 2011/01/05. eng. [PubMed: 21200000]

5. Powers BJ, Olsen MK, Smith VA, Woolson RF, Bosworth HB, Oddone EZ. Measuring blood pressure for decision making and quality reporting: where and how many measures? Ann Intern Med. Jun 21; 2011 154(12):781-8. W-289-90. PubMed PMID: 21690592. Epub 2011/06/22. eng. [PubMed: 21690592]

6. Cuffe RL, Howard SC, Algra A, Warlow CP, Rothwell PM. Medium-term variability of blood pressure and potential underdiagnosis of hypertension in patients with previous transient ischemic attack or minor stroke. Stroke. Nov; 2006 37(11):2776-83. PubMed PMID: 17008634. Epub 2006/09/30. eng. [PubMed: 17008634]

7. National Center for Health Statistics. Plan and operation of the third National Health and Nutrition Examination survey, 1988-1994. Series 1: programs and collection procedures. Vital Health Stat 1. 1994:94-1308.

8. Bland JM, Altman DG. Statistical methods for assessing agreement between two methods of clinical measurement. Lancet. Feb 8; 1986 1(8476):307-10. PubMed PMID: 2868172. Epub 1986/02/08. eng. [PubMed: 2868172]

9. Framingham Heart Study. [Accessed September 4, 2012] Hard Coronary Heart Disease (10-year risk). Framingham Heart Study Web site. 2012. Available at: http:// www.framinghamheartstudy.org/risk/hrdcoronary.html.

10. Grundy SM, Cleeman JI, Merz CN, Brewer HB Jr. Clark LT, Hunninghake DB, et al. Implications of recent clinical trials for the National Cholesterol Education Program Adult Treatment Panel III guidelines. Circulation. Jul 13; 2004 110(2):227-39. PubMed PMID: 15249516. Epub 2004/07/14. eng. [PubMed: 15249516]

11. Shimbo D, Newman JD, Aragaki AK, Lamonte MJ, Bavry AA, Allison M, et al. Association Between Annual Visit-to-Visit Blood Pressure Variability and Stroke in Postmenopausal Women: Data From the Women's Health Initiative. Hypertension. Sep; 2012 60(3):625-30. PubMed PMID: 22753206. Pubmed Central PMCID: 3427141. Epub 2012/07/04. eng. [PubMed: 22753206]

12. Berger JS, Jordan CO, Lloyd-Jones D, Blumenthal RS. Screening for cardiovascular risk in asymptomatic patients. J Am Coll Cardiol. Mar 23; 2010 55(12):1169-77. PubMed PMID: 20298922. Epub 2010/03/20. eng. [PubMed: 20298922]

13. Hippisley-Cox J, Coupland C, Vinogradova Y, Robson J, Minhas R, Sheikh A, et al. Predicting cardiovascular risk in England and Wales: prospective derivation and validation of QRISK2. BMJ (Clinical research ed). Jun 28; 2008 336(7659):1475-82. PubMed PMID: 18573856. Pubmed Central PMCID: PMC2440904. Epub 2008/06/25. eng.

14. Ridker P, Buring JE, Rifai N, Cook NR. Development and validation of improved algorithms for the assessment of global cardiovascular risk in women: The reynolds risk score. JAMA. 2007; 297(6):611-9. [PubMed: 17299196]

15. Perk J, De Backer G, Gohlke H, Graham I, Reiner Z, Verschuren M, et al. European Guidelines on cardiovascular disease prevention in clinical practice (version 2012). The Fifth Joint Task Force of 
the European Society of Cardiology and Other Societies on Cardiovascular Disease Prevention in Clinical Practice (constituted by representatives of nine societies and by invited experts). Developed with the special contribution of the European Association for Cardiovascular Prevention \& Rehabilitation (EACPR). Eur Heart J. Jul; 2012 33(13):1635-701. PubMed PMID: 22555213. Epub 2012/05/05. eng. [PubMed: 22555213]

16. Bower JK, Lazo M, Juraschek SP, Selvin E. WIthin-person variability in high-sensitivity c-reactive protein. Archives of Internal Medicine. 2012; 172(19):1519-21. [PubMed: 22945505]

17. Lloyd-Jones DM. Cardiovascular Risk Prediction. Circulation. Apr 20; 2010 121(15):1768-77. 2010. [PubMed: 20404268]

18. Walsh MN, Bove AA, Cross RR, Ferdinand KC, Forman DE, Freeman AM, et al. ACCF 2012 health policy statement on patient-centered care in cardiovascular medicine: a report of the American College of Cardiology Foundation Clinical Quality Committee. J Am Coll Cardiol. Jun 5; 2012 59(23):2125-43. PubMed PMID: 22591882. Epub 2012/05/18. eng. [PubMed: 22591882]

19. Kent DM, Shah ND. Risk models and patient-centered evidence: should physicians expect one right answer? JAMA. Apr 18; 2012 307(15):1585-6. PubMed PMID: 22511683. Epub 2012/04/19. eng. [PubMed: 22511683]

20. Ford ES, Giles WH, Mokdad AH. The distribution of 10-Year risk for coronary heart disease among US adults: findings from the National Health and Nutrition Examination Survey III. J Am Coll Cardiol. May 19; 2004 43(10):1791-6. PubMed PMID: 15145101. Epub 2004/05/18. eng. [PubMed: 15145101]

21. Levitan EB, Kaciroti N, Oparil S, Julius S, Muntner P. Blood Pressure Measurement Device, Number and Timing of Visits, and Intra-Individual Visit-to-Visit Variability of Blood Pressure. J Clin Hypertens (Greenwich). Nov; 2012 14(11):744-50. PubMed PMID: 23126345. Epub 2012/11/07. Eng. [PubMed: 23126345] 


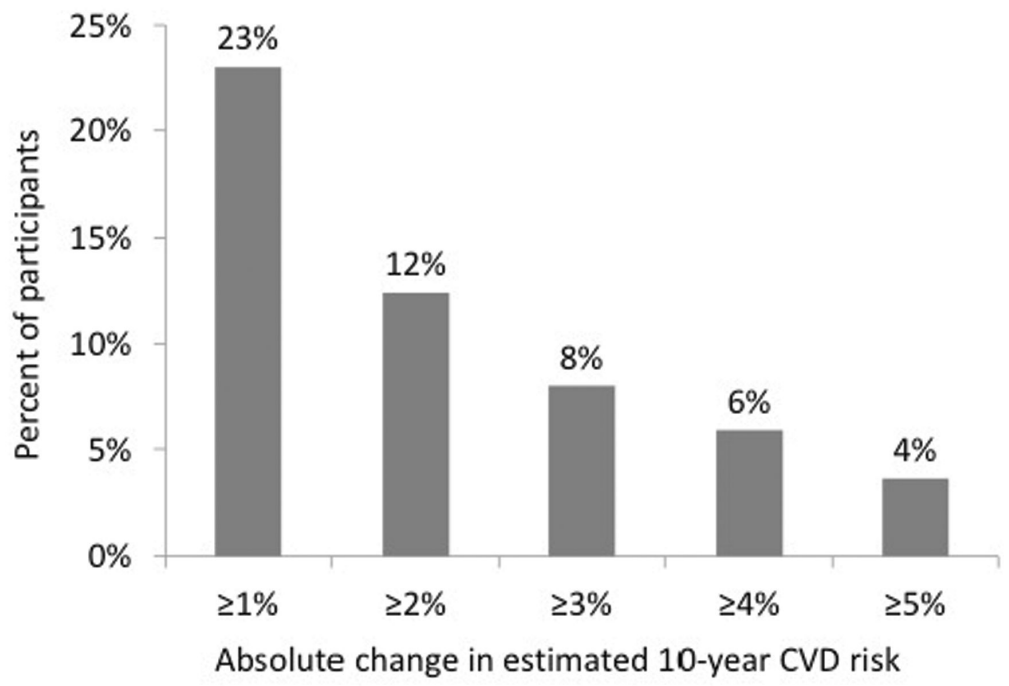

Figure 1. Percentage of participants with an absolute change in estimated 10-year cardiovascular disease (CVD) risk $\geq 1 \%$ to $\geq 5 \%$ resulting from change in systolic blood pressure across two clinic visits.

Absolute change in estimated 10-year CVD risk is calculated as the absolute difference between Pooled Cohort Equations derived risk estimates determined at clinic visit 1 and clinic visit 2, using systolic blood pressure measured at each visit while holding all other variables constant. 


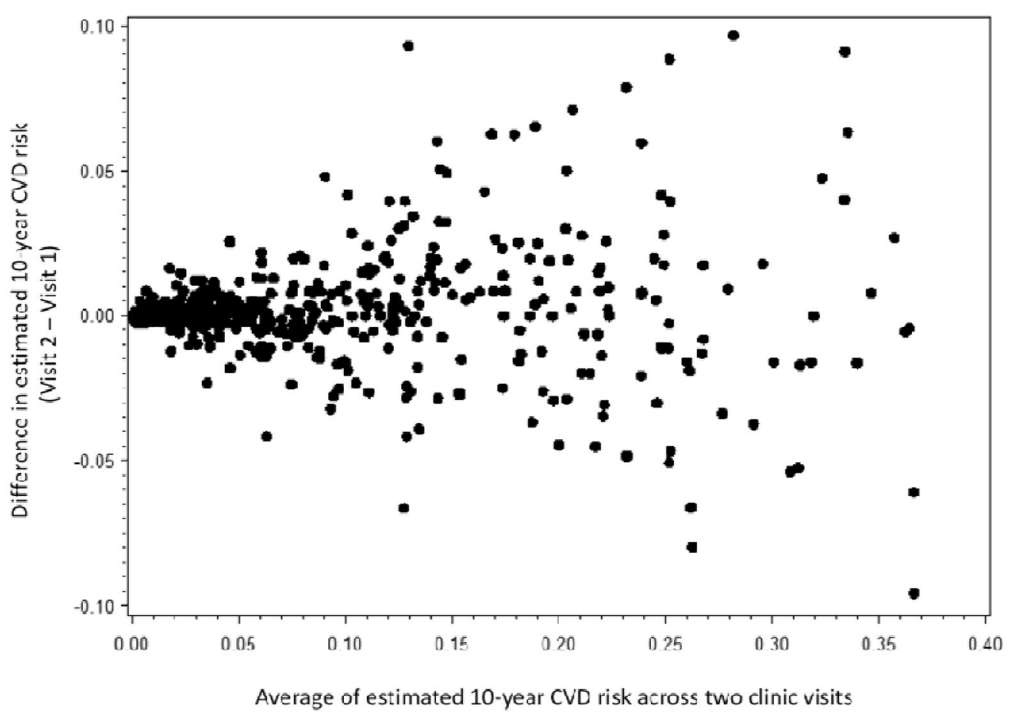

Figure 2.

Bland-Altman plot for comparison of estimated 10-year cardiovascular disease (CVD) risk calculated using the Pooled Cohort Equations at two clinic visits. 


\section{Table 1}

Characteristics of NHANES III participants who had blood pressure measurements at two clinic visits.

\begin{tabular}{|l|c|}
\hline Characteristic & N=808 \\
\hline Age, years & $46.1(17.2)$ \\
\hline Male sex & $47.3 \%$ \\
\hline Race-ethnicity & \\
Non-Hispanic white & $42.2 \%$ \\
Non-Hispanic black & $27.9 \%$ \\
Mexican American & $25.7 \%$ \\
Other & $4.2 \%$ \\
\hline Current smoking & $29.2 \%$ \\
\hline Total cholesterol, mmol/L & $5.31(1.08)$ \\
\hline HDL-cholesterol, mmol/L & $1.31(0.39)$ \\
\hline On antihypertensive treatment & $14.5 \%$ \\
\hline
\end{tabular}

HDL, high-density lipoprotein; numbers in table are mean (standard deviation) or percentage. 
Table 2

Systolic and diastolic blood pressure and Pooled Cohort Equations estimated 10-year cardiovascular disease risk at each NHANES III visit.

\begin{tabular}{|c|c|c|c|c|c|}
\hline & \multicolumn{5}{|c|}{ Percentile } \\
\hline & $1^{\text {st }}$ & $25^{\text {th }}$ & $50^{\text {th }}$ & $75^{\text {th }}$ & $99^{\text {th }}$ \\
\hline \multicolumn{6}{|l|}{ Clinic Visit $1 \mathrm{BP}, \mathrm{mmHg}$} \\
\hline Systolic & 91 & 108 & 118 & 131 & 180 \\
\hline Diastolic & 47 & 65 & 73 & 80 & 100 \\
\hline \multicolumn{6}{|l|}{ Clinic Visit 2 BP, mmHg } \\
\hline Systolic & 90 & 107 & 117 & 129 & 174 \\
\hline Diastolic & 46 & 65 & 72 & 79 & 97 \\
\hline \multicolumn{6}{|l|}{ Difference in $\mathrm{BP}, \mathrm{mmHg}$ (clinic visit 2 - clinic visit 1 ) } \\
\hline SBP & -23 & -7 & -1 & 4 & 32 \\
\hline DBP & -17 & -5 & 0 & 4 & 21 \\
\hline \multicolumn{6}{|l|}{ Estimated 10-year CVD risk ${ }^{*}, \%$} \\
\hline Clinic Visit 1 SBP & $<0.1 \%$ & $0.5 \%$ & $2.5 \%$ & $10.0 \%$ & $49.9 \%$ \\
\hline Clinic Visit 2 SBP & $<0.1 \%$ & $0.5 \%$ & $2.4 \%$ & $10.0 \%$ & $45.8 \%$ \\
\hline Differences in estimated 10 -year CVD risk, \% (clinic visit 1 - clinic visit 2 ) & $-5.2 \%$ & $-0.1 \%$ & $0.0 \%$ & $0.3 \%$ & $7.1 \%$ \\
\hline
\end{tabular}

BP: blood pressure; SBP, systolic blood pressure; DBP, diastolic blood pressure; CVD: cardiovascular disease.

Estimated 10-year CVD risk are calculated from the Pooled Cohort Equations recommended by the American College of Cardiology Foundation / American Heart Association 2013 Guideline on the Assessment of Cardiovascular Risk. 


\section{Table 3}

Percent of participants whose estimated 10-year cardiovascular disease (CVD) risk was $\mathbf{3} \%$ different due to systolic blood pressure variability between two clinic visits and odds ratios for $23 \%$ difference by categories of systolic blood pressure (top panel) and diastolic blood pressure (bottom panel).

\begin{tabular}{|c|c|c|c|}
\hline & \multicolumn{3}{|c|}{ Systolic blood pressure from Visit $1, \mathrm{~mm} \mathrm{Hg}$} \\
\hline & $<130(\mathrm{n}=579)$ & $130-149(n=154)$ & $>\geq 150(\mathrm{n}=75)$ \\
\hline \multirow[t]{2}{*}{$\%$ with $>3 \%$ change in 10 -year CVD risk } & $2.9 \%$ & $13.6 \%$ & $36.0 \%$ \\
\hline & \multicolumn{3}{|c|}{ Odds ratio $(95 \% \mathrm{Cl})$ for reclassification } \\
\hline Model 1 & 1 (ref) & $1.76(0.80-3.84)$ & $4.72(2.06-10.8)$ \\
\hline \multirow[t]{3}{*}{ Model $2^{\dagger}$} & 1 (ref) & $1.86(0.84-4.12)$ & $5.60(2.23-14.1)$ \\
\hline & \multicolumn{3}{|c|}{ Diastolic blood pressure from Visit $1, \mathrm{~mm} \mathrm{Hg}$} \\
\hline & $<75(\mathrm{n}=457)$ & $75-84(n=222)$ & $\geq 85(\mathrm{n}=129)$ \\
\hline \multirow[t]{2}{*}{$\%$ with $>3 \%$ change in 10 -year CVD risk } & $7.4 \%$ & $9.9 \%$ & $7.0 \%$ \\
\hline & \multicolumn{3}{|c|}{ Odds ratio $(95 \% \mathrm{Cl})$ for reclassification } \\
\hline Model 1 & 1 (ref) & $1.35(0.68-2.69)$ & $1.41(0.55-3.66)$ \\
\hline Model $2^{\dagger \dagger}$ & 1 (ref) & $1.00(0.48-2.06)$ & $0.60(0.21-1.78)$ \\
\hline
\end{tabular}

Model 1 includes adjustment for age, gender, and race-ethnicity

${ }^{\dagger}$ Model 2 includes adjustment for age, gender, race-ethnicity, and diastolic blood pressure

${ }^{\dagger}$ Model 2 includes adjustment for age, gender, race-ethnicity, and systolic blood pressure 


\section{Table 4}

Reclassification of estimated 10-year cardiovascular disease (CVD) risk of NHANES III participants due to change in systolic blood pressure across two clinic visits.

\begin{tabular}{|c|c|c|c|c|}
\hline & \multicolumn{4}{|c|}{ Estimated 10-year CVD risk at Visit 1 } \\
\hline Estimated 10-year CVD risk at Visit 2 & $<\mathbf{5 \%}$ & $\mathbf{5}$ to $<\mathbf{7 . 5 \%}$ & $\mathbf{7 . 5 \%}$ to $<\mathbf{1 0 \%}$ & $\mathbf{1 0 \%}$ \\
\hline $\mathbf{5} \%$ & $494(98.8 \%)$ & $7(12.1 \%)$ & $0(0 \%)$ & $0(0 \%)$ \\
\hline $\mathbf{5}$ to $<\mathbf{7 . 5 \%}$ & $5(1.0 \%)$ & $45(77.6 \%)$ & $6(12.5 \%)$ & $1(0.5 \%)$ \\
\hline $\mathbf{7 . 5 \%}$ to $<\mathbf{1 0 \%}$ & $1(0.2 \%)$ & $6(10.3 \%)$ & $32(66.7 \%)$ & $8(4.0 \%)$ \\
\hline$\geq \mathbf{1 0 \%}$ & $0(0 \%)$ & $0(0 \%)$ & $10(20.8 \%)$ & $193(95.5 \%)$ \\
\hline Total & $500(61.9 \%)$ & $58(7.2 \%)$ & $48(5.9 \%)$ & $202(25.0 \%)$ \\
\hline
\end{tabular}

CVD, cardiovascular disease; numbers in table are $\mathrm{n}(\%)$.

*Percentages are calculated per column using the total number of participants in each Clinic Visit 1 estimated 10-year CVD risk category as denominator; may not add up to $100 \%$ due to rounding 


\section{Table 5}

Reclassification of Framingham equation estimated 10-year coronary heart disease (CHD) risk in NHANES III participants due to change in systolic blood pressure across two clinic visits.

\begin{tabular}{|c|c|c|c|}
\hline & \multicolumn{3}{|c|}{ Estimated 10-year CHD risk at Visit 1 } \\
\hline Estimated 10-year CHD risk at Visit 2 & $<\mathbf{1 0 \%}$ & $\mathbf{1 0 - 2 0 \%}$ & $>\mathbf{2 0 \%}$ \\
\hline $\mathbf{1 0 \%}$ & $633(98.9 \%)$ & $14(10.9 \%)$ & $0(0 \%)$ \\
\hline $\mathbf{1 0 - 2 0 \%}$ & $7(1.1 \%)$ & $110(85.9 \%)$ & $10(25.0 \%)$ \\
\hline$>\mathbf{2 0 \%}$ & $0(0 \%)$ & $4(3.1 \%)$ & $30(75.0 \%)$ \\
\hline Total & $640(100 \%)$ & $128(100 \%)$ & $40(100 \%)$ \\
\hline
\end{tabular}

CHD, coronary heart disease; numbers in table are $\mathrm{n}(\%)$.

*Percentages are calculated per column using the total number of participants in each Clinic Visit 1 estimated 10-year CHD risk category as denominator; may not add up to $100 \%$ due to rounding. 


\title{
Table 6
}

Examples of NHANES III participants whose Pooled Cohort Equations estimated 10-year CVD risk were reclassified due to change in systolic blood pressure across two clinic visits.

\begin{abstract}
Participant A
A 41 year old African-American woman with total cholesterol of $4.27 \mathrm{mmol} / \mathrm{L}, \mathrm{HDL}$ of $1.03 \mathrm{mmol} / \mathrm{L}$, and SBP of $\mathbf{1 4 5} \mathbf{~ m m ~} \mathbf{~ H g}$ at clinic visit 1 . She is a smoker and is not on medication treatment for hypertension. Her Pooled Cohort Equations estimated 10-year CVD risk is 4.2\%. At clinic visit 2, her SBP is $\mathbf{1 6 6} \mathbf{~ m m ~ H g}$, which changes her estimated 10-year CVD risk to $\mathbf{8 . 4 \%}$.

\section{Participant B}

A 46 year old nonHispanic white man with total cholesterol of $6.52 \mathrm{mmol} / \mathrm{L}$, HDL of $0.78 \mathrm{mmol} / \mathrm{L}$, and SBP of $149 \mathrm{~mm} \mathbf{~ H g}$ at clinic visit 1 . He does not smoke and does not take any antihypertensive medications. His Pooled Cohort Equations estimated 10-year CVD risk is 7.9\%. At clinic visit 2, his SBP is $\mathbf{1 4 0 ~} \mathbf{~ m m ~ H g}$, which changes his estimated 10-year CVD risk to $\mathbf{7 . 1 \%}$.
\end{abstract}

CVD: cardiovascular disease; HDL, high-density lipoprotein; SBP, systolic blood pressure. 